\title{
Una patología muy infrecuente: ganglioneuromatosis intestinal. Reporte de un caso
}

\author{
Marcos Leites, ${ }^{1}$ Alejandra Arriola, ${ }^{1}$ Yéssica Pontét, ${ }^{1}$ Iván Trochansky, ${ }^{2}$ Marta Otero, ${ }^{3}$ \\ Carolina Olano ${ }^{1}$ \\ ${ }^{1}$ Clínica de Gastroenterología Prof. Dr. Henry Cohen. \\ ${ }^{2}$ Clínica Quirúrgica Prof. Dr. Roberto Valiñas. \\ ${ }^{3}$ Cátedra y Departamento de Anatomía Patológica Prof. Dr. Dardo Centurión.
}

Hospital de Clínicas Dr. Manuel Quintela. Montevideo, Uruguay.

Acta Gastroenterol Latinoam 2020;50(2):205-209

Recibido: 27/02/2019 / Aceptado: 15/05/2019 / Publicado online: 29/06/2020

\section{Resumen}

La ganglioneuromatosis intestinal es una patología infrecuente, cuya presentación en adultos es excepcional. Clásicamente se asocia con el sindrome de neoplasias endócrinas múltiples de tipo IIb y con la neurofibromatosis de Von Recklinghausen. Desde la histología se la caracteriza por la hiperplasia de los plexos intramurales del tubo digestivo y de las fibras nerviosas entéricas, lo que suele traducirse de forma clinica en cuadros de dolor abdominal, distensión y alteraciones del tránsito intestinal. Se presenta el caso de una paciente de 38 años, portadora del síndrome de neoplasias endócrinas múltiples tipo IIb, que se manifestó con reiterados cuadros oclusivos y suboclusivos, requiriendo en la evolución una colectomia parcial. A través del estudio anatomopatológico de la pieza quirúrgica se realizó el diagnóstico de ganglioneuromatosis intestinal.

Palabras claves. Ganglioneuromatosis, enfermedad de Hirschsprung, neoplasia endócrina múltiple, tiroidectomía.

\section{A very unusual pathology: intestinal ganglioneuromatosis. Case report}

\section{Summary}

Intestinal ganglioneuromatosis is an unusual pathology, especially in adults. It is associated with multiple endocrine neoplasia type IIb and Von Recklinghausen's neurofibromatosis. Histologically, it is characterized by hyperplasia of the intramural plexuses of the gastrointestinal tract and enteric nerve fibres. Clinical manifestations include abdominal pain, distension and changes in bowel habits. We report the case of a 38-year-old patient, with a multiple endocrine neoplasia syndrome type IIb, presenting with repeated partial or complete intestinal obstruction that requires partial colectomy. Pathology of the resected colon revealed intestinal ganglioneuromatosis.

Key words. Ganglioneuromatosis, Hirschsprung's disease, multiple endocrine neoplasia, thyroidectomy.
Correspondencia: Marcos Leites

Eduardo Victor Haédo 2322. Montevideo, Uruguay

Tel.: (+598) 91231322

Correo electrónico: mleitesmadera@gmail.com

\author{
Abreviaturas \\ GNM: Ganglioneuromatosis. \\ MEN IIb: Neoplasias endócrinas múltiples tipo IIb. \\ VRN: Von Recklinghausen neurofibromatosis. \\ TC: Tomografia computada. \\ VCC: Videocolonoscopía. \\ VGC: Videogastroscopía.
}




\section{Introducción}

La GNM intestinal es una enfermedad muy infrecuente caracterizada por la hiperplasia de plexos intramurales del tubo digestivo y de fibras nerviosas entéricas. ${ }^{1,2}$ Las células de Schwann y las células ganglionares son las más afectadas, presentando hiperplasia difusa. La etiopatogenia del trastorno no es conocida. Se postula la participación de factores de crecimiento nervioso que estimularían o dejarían de inhibir la proliferación celular, conduciendo al desarrollo de los ganglioneuromas. ${ }^{3,4}$ Afecta fundamentalmente a niños, en quienes suele plantearse inicialmente el diagnóstico de enfermedad de Hirschsprung y, por medio de la anatomía patológica, se arriba al diagnóstico de GNM., 4

Se describen dos variantes de presentación. La presentación mucosa se caracteriza por la hiperplasia de los nervios de la mucosa intestinal y del plexo submucoso. La presentación transmural se caracteriza por la hiperplasia nerviosa transmural con el compromiso predominante del plexo mientérico. ${ }^{1,2}$

Algunos autores afirman que la forma transmural predomina en la edad pediátrica y la presentación mucosa es la más frecuente en la adultez. ${ }^{1,2}$

Puede ocurrir como una entidad aislada, pero habitualmente se la asocia a otras patologías como: síndrome de MEN IIb, VRN, poliposis adenomatosa no familiar $\mathrm{y}$, de manera menos establecida, con adenocarcinoma del intestino delgado y colon. ${ }^{1-3,5}$

La importancia de su diagnóstico radica, en gran parte, en la búsqueda de neoplasias asociadas. En los casos publicados sobre niños, los síntomas pseudo-Hirschsprung preceden al diagnóstico de carcinoma medular de tiroi$\mathrm{des}^{2,4} \mathrm{y}$, su presencia en adultos, se asocia probablemente a un mayor riesgo de neoplasias intestinales. ${ }^{1,5}$

Puede afectar a cualquier segmento del tracto gastrointestinal señalándose al íleon, colon y apéndice como las regiones perjudicadas con mayor frecuencia., ${ }^{2,5}$

Su presentación en el adulto es excepcional y las manifestaciones clínicas dependen del sector del tubo digestivo damnificado, la extensión de la afectación y su repercusión sobre la motilidad intestinal. Los síntomas más frecuentes son: dolor abdominal del tipo cólico, distensión, alteración en el hábito defecatorio (constipación crónica con episodios intermitentes de diarrea) y cuadros de oclusión/ suboclusión del intestino. Estos últimos se pueden explicar por estenosis o por cambios segmentarios en la motilidad intestinal. Con menor frecuencia puede presentarse como una hemorragia digestiva oculta secundaria a una ulceración o erosiones de la mucosa, siendo posible también que el paciente permanezca asintomático por décadas. ${ }^{5}$
El diagnóstico es anatomopatológico y se puede alcanzar a partir de muestras de biopsias obtenidas por métodos endoscópicos o del análisis de piezas quirúrgicas. ${ }^{1-3,5}$

Debido a la baja frecuencia de esta patología, no se plantea de forma habitual como un diagnóstico diferencial en cuadros suboclusivos, siendo necesario un alto índice de sospecha para evitar el retraso en el diagnóstico e intervenciones inapropiadas.

\section{Caso clínico}

Mujer de 38 años, con antecedentes de colecistectomía por laparotomía, hipertensión arterial, síndrome de MEN IIb manifestado por carcinoma medular de tiroides y feocromocitoma.

De sus antecedentes familiares se destaca: dos hermanos fallecidos por carcinoma medular de tiroides, una hija de 17 años con síndrome de MEN IIb y un hijo de 18 años tiroidectomizado. No contamos con datos sobre su indicación.

En los 4 años previos al diagnóstico presentó episodios reiterados de oclusión/ suboclusión intestinal. Durante uno de ellos se le realizó una TC de abdomen y se planteó una oclusión por vólvulo de intestino delgado. Se realiza laparotomía de urgencia, en la que se constatan bridas y se degravita manualmente.

Ella reitera cuadros clínicos similares que conducen a una nueva laparotomía de urgencia en la que se constata un megacolon. Se realiza una colectomía derecha con restitución del tránsito mediante íleo-sigmoideo anastomosis.

A nivel macroscópico, la pieza quirúrgica presenta mucosa con disminución del número de pliegues principalmente en el colon transverso, luz colónica de hasta $110 \mathrm{~mm}$ de diámetro y la pared adelgazada (Figura 1).

Microscópicamente presenta el epitelio de revestimiento con áreas de esfacelo y discretos cambios regenerativos. En el nivel de la capa muscular y subserosa se observan numerosos nódulos bien delimitados compuestos por células ganglionares en un fondo neurofibrilar. La subserosa muestra una hiperplasia e hipertrofia de filetes nerviosos (Figura 2). Se realiza el diagnóstico de ganglioneuromatosis colónica, con cabos de resección comprometidos por el proceso, sin elementos de malignidad.

$\mathrm{Al}$ año, se reitera episodio de oclusión.

Se realiza una TC de abdomen que informa una gran distensión de las asas intestinales (Figura 3), punto de transición a nivel de sigmoides con signo del remolino en relación al vólvulo. Se degravita mediante VCC. 
Figura 1. Pieza quirúrgica: se observa marcada dilatación de la luz colónica y adelgazamiento parietal.

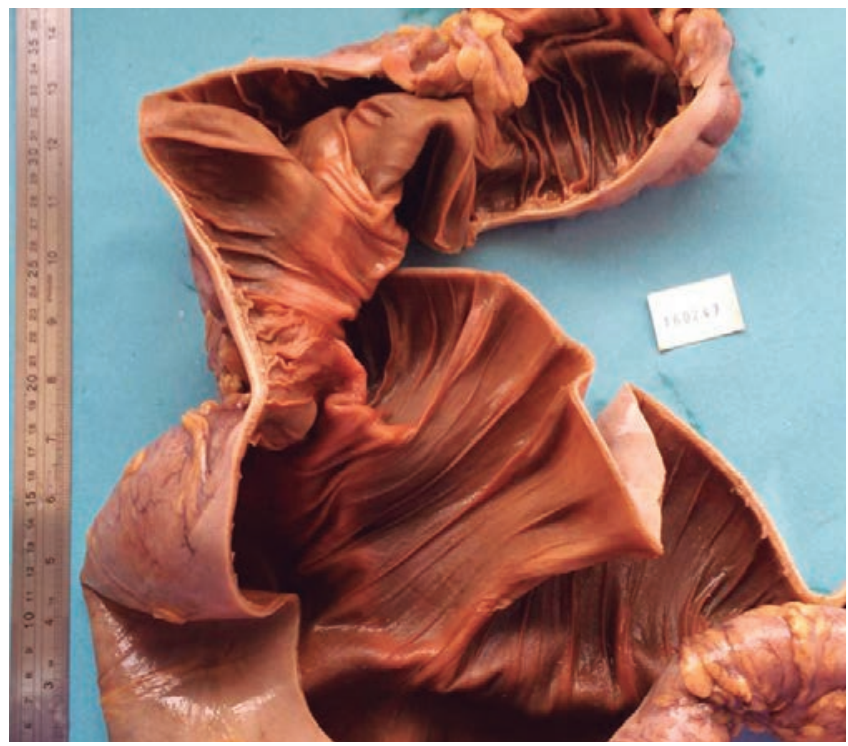

Figura 2. Microscopía (HङE 40X): lesión intramural caracterizada por la expansión hiperplásica de los elementos del plexo mientérico, nodular o irregular (flecha).

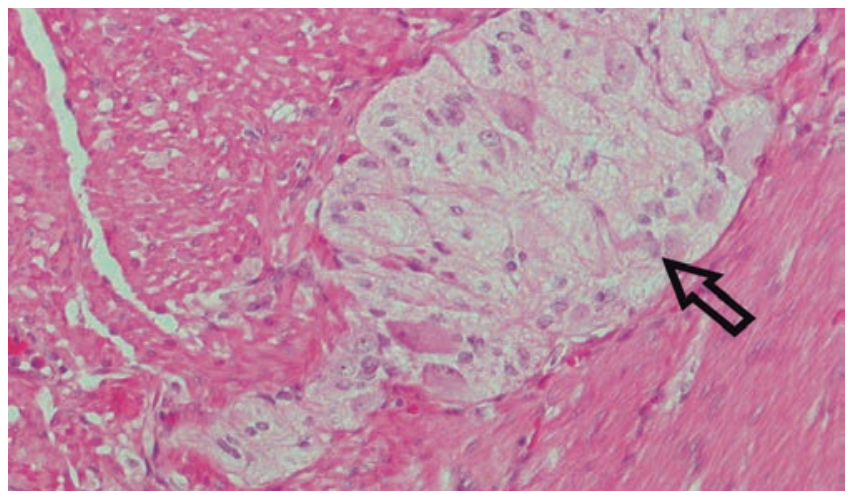

Figura 3. Tomografía computada: se observa gran dilatación de las asas intestinales con niveles hidroaéreos.

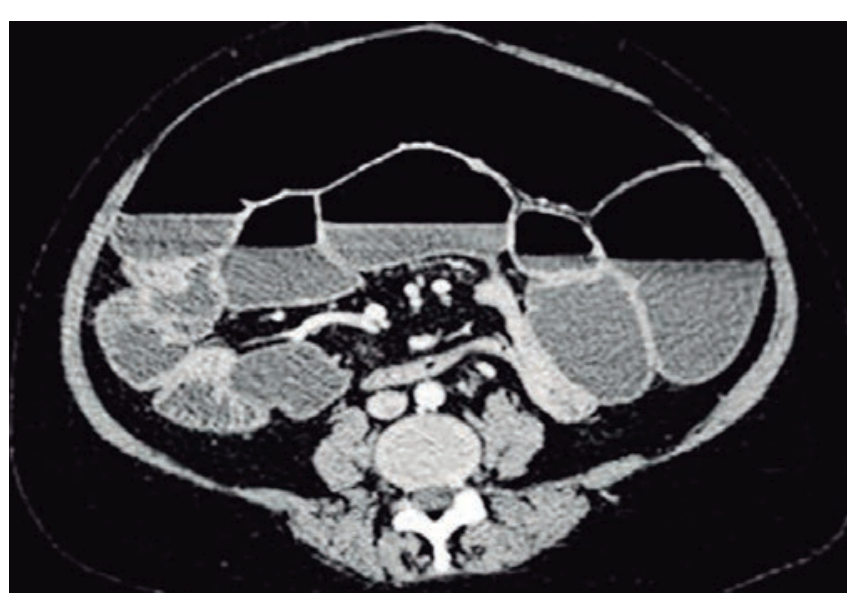

Se realiza un nuevo estudio endoscópico con la finalidad de valorar la extensión de la enfermedad. La anatomía patológica destaca mucosa colónica e ileal con leves elementos inflamatorios inespecíficos, sin displasia ni neoplasia. Al revisar la pieza quirúrgica se evidencia una afectación ileal con cabo de resección proximal comprometido (Figura 4).

La inmunohistoquímica es positiva para S100 (Figura 5) lo que apoya el diagnóstico de GNM intestinal.

Figura 4. Microscopia (H\&E 10X): Cabo de la resección proximal, pared de ileon con hiperplasia de los plexos mientéricos (flechas).

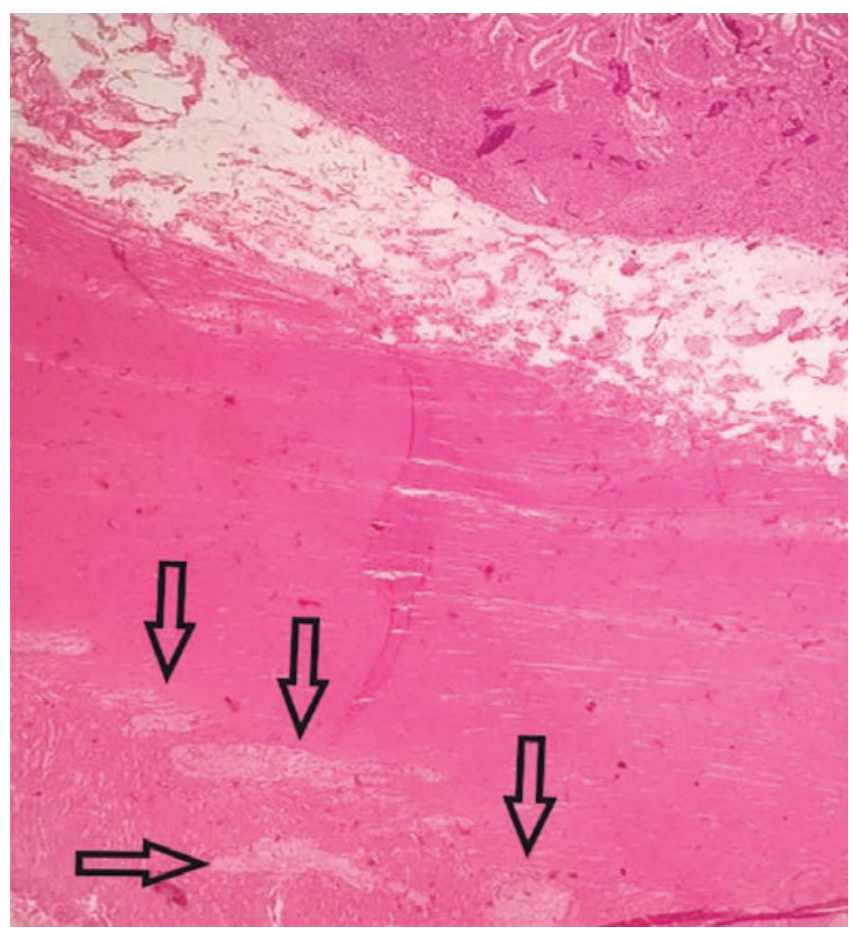

Figura 5. Inmunohistoquimica: la inmunomarcación con proteina S100 evidencia la estirpe neural de la lesión.

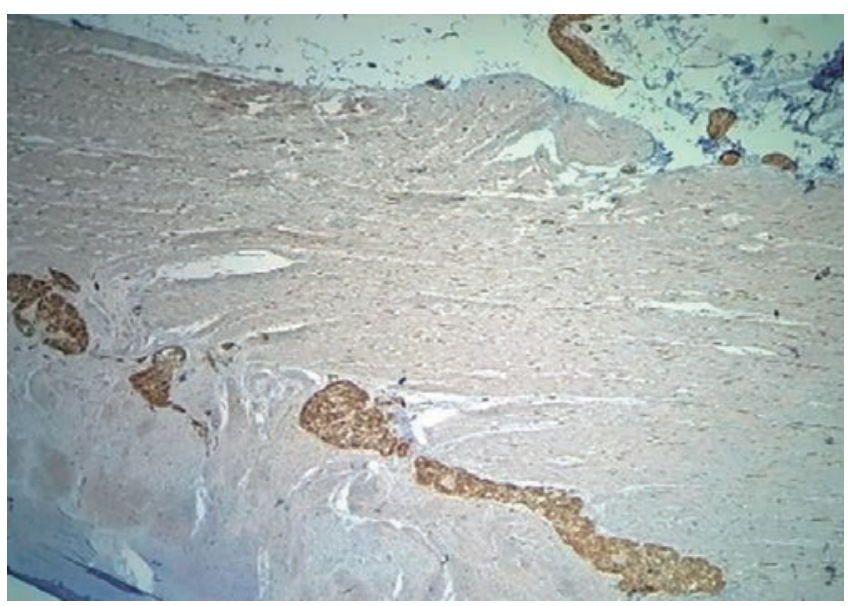


En la evolución, la paciente alterna períodos de remisión sintomática con nuevos episodios oclusivos/suboclusivos que se resuelven con degravitación mediante una sonda rectal.

Se decide no realizar una resección quirúrgica por la elevada probabilidad de aparición de síndrome de intestino corto. Se le indica un tratamiento médico en base a rifaximina lográndose una respuesta clínica satisfactoria con la regularización parcial del tránsito intestinal, una disminución de la distensión abdominal y la ausencia de nuevos episodios suboclusivos.

\section{Discusión}

Se presenta el caso de una paciente portadora de GNM intestinal, con múltiples episodios oclusivos y suboclusivos que requirieron frecuentes ingresos hospitalarios con una resolución quirúrgica, endoscópica o degravitación mediante una sonda rectal.

Se trata de una patología de presentación excepcional en la edad adulta, por lo que los datos en la literatura son escasos, desconociéndose con exactitud el tratamiento, seguimiento y pronóstico de estos pacientes.

La información disponible en la actualidad proviene de los reportes y de una serie de casos. Chambonnière ${ }^{1}$ en el año 2003 reportó 10 casos de GNM intestinal presentados en adultos. A partir de esta publicación se reconocieron algunas peculiaridades de esta entidad que la distinguen de la presentación de la GNM intestinal en la infancia. En dicha serie, la forma de presentación más frecuente es la mucosa (90\%) con la afectación exclusiva del colon en todos los casos estudiados. Sin embargo, la afectación puede ser variable. Así un caso reportado en Argentina evidenció la afectación transmural con compromiso desde estómago al colon inclusive. ${ }^{2}$ Fernandes 5 publicó un caso con una afectación exclusivamente ileal en un paciente con una hemorragia digestiva oculta. Finalmente, dos casos notificados en Corea demuestran una afectación transmural sin compromiso del íleon, afectando rectosigmoides y colon respectivamente. ${ }^{3}$ En contraposición, la literatura sobre casos infantiles evidencia una afectación persistente o casi constante del íleon. ${ }^{1,6}$

En el caso reportado, los múltiples episodios de oclusión intestinal conducen a los planteos de cierre por bridas y vólvulo de delgado, arribando, luego de 4 años, al diagnóstico de GNM con el compromiso transmural del íleon y del colon.

La información descripta en la literatura sobre los hallazgos en la pieza quirúrgica destaca la presencia de pólipos con una mucosa de aspecto normal entre ellos, y una apariencia más delgada y desprovista de pliegues. Se describe también la presencia de tumores y megacolon sin un obstáculo orgánico. ${ }^{1,5}$ En el caso de la paciente, se describe la presencia del megacolon sin un obstáculo orgánico identificable, la mucosa de aspecto normal con una disminución del número de pliegues y la pared intestinal adelgazada.

La evidencia actual señala que la endoscopia puede demostrar una espasticidad inusual en el colon, siendo frecuente la presencia de pólipos únicos o múltiples de hasta $30 \mathrm{~mm} .{ }^{1,5}$ En la mayoría de los pacientes, la mucosa entre los pólipos es de aspecto normal, describiéndose en algunos casos la presencia de un dolicocolon o un megacolon.1 En esta paciente, en el primer episodio oclusivo, se le realizó una VCC de urgencia con una mala preparación, que no evidencia alteraciones.

Un punto interesante es el rendimiento del diagnóstico en las biopsias obtenidas a través de estudios endoscópicos. En la serie de Chambonnière ${ }^{1}$ las biopsias endoscópicas posibilitan el diagnóstico solo en la mitad de los pacientes y en los demás se obtiene mediante la pieza quirúrgica. Esto probablemente se deba al compromiso mural presente.

En muchos casos la tinción clásica de $\mathrm{H} \& \mathrm{E}$ suele ser suficiente para detectar las alteraciones histológicas, siendo las técnicas de inmunohistoquímica de gran ayuda para confirmar el origen nervioso de la lesión (anticuerpos contra la proteína S100). La detección de la proteína Tau o c-Ret puede ser útil para arribar al diagnóstico y para la diferenciación con la neurofibromatosis, en la que no existe proliferación de células ganglionares. ${ }^{1,3,5}$

Con respecto a la terapéutica, no existen tratamientos médicos que permitan el manejo sintomático de estos pacientes y, menos aún, curativos, por lo que su resolución es quirúrgica. ${ }^{2,3,5}$ Se plantea la cirugía con la resección del segmento afectado cuando se produce una oclusión intestinal o cuando se sospecha una enfermedad neoplásica. ${ }^{5}$ Luego de la resección, se debe evaluar si la capacidad absortiva restante es suficiente, ya que las resecciones amplias pueden condicionar la aparición del síndrome del intestino corto. ${ }^{2,5}$ No se encuentran en la literatura datos reportados sobre trasplantes intestinales en esta patología, seguramente por su infrecuencia.

$\mathrm{Al}$ igual que la enfermedad de Hirschsprung y de Crohn, la GNM ocasiona alteraciones de la motilidad intestinal, estenosis y el síndrome suboclusivo que requieren una resección quirúrgica. ${ }^{2,7,8}$

En el caso reportado, se comprueba la afectación del íleon distal y del colon, no pudiéndose descartar el compromiso de los sectores más proximales. Es por tal razón que no se plantea una resección pretendidamente curati- 
va, ya que esto puede conducir al síndrome del intestino corto, sin tener la certeza de lograr la resolución clínica. Se plantea una conducta expectante y el tratamiento médico con rifaximina en forma continua para mejorar los síntomas (dolor abdominal y diarrea por sobrecrecimiento bacteriano), lográndose una respuesta clínica satisfactoria.

Debido a lo infrecuente de esta patología y a su potencial riesgo neoplásico se plantean muchas interrogantes acerca de cuál es la mejor estrategia de seguimiento, la herramienta de diagnóstico más adecuada y los intervalos de tiempo a adoptar.

\section{Conclusión}

La GNM es una patología muy infrecuente, especialmente en adultos. La carencia de datos bibliográficos determina múltiples interrogantes acerca de cuál es el mejor tratamiento, su pronóstico y las pautas de seguimiento.

Sostén Financiero. Para la realización del presente trabajo no se recibieron aportes financieros.

\section{Referencias}

1. Chambonnière ML, Porcheron J, Scoazec JY, Audigier JC, Mosnier JF. Intestinal ganglioneuromatosis diagnosed in adult patients. Gastroenterol Clin Biol 2003; 27: 219-224.

2. Godoy N, Parodi R, Díaz M, Valenti J, Carlson D, Greca A. Ganglioneuromatosis difusa: comunicación de una patología infrecuente y revisión de la literatura. Acta Gastroenterol Latinoam 2010; 40: 151-155.

3. Kim TJ, Lim H, Kang HS, Moon S, Kim J, Park C, Kwon N, Lee $\mathrm{BH}$. Diffuse ganglioneuromatosis of the colon presenting as a large subepithelial tumor in adults: report of two cases. Korean J Gastroenterol 2015; 66 (2): 111-115.

4. Smith VV, Eng C, Milla PJ. Intestinal ganglioneuromatosis and multiple endocrine neoplasia type 2B: implications for treatment. Gut 1999; 45: 143-146.

5. Fernandes A, Ferreira A, Serra P, Carvalho L. Intestinal ganglioneuromatosis: an unusual aetiology for occult gastrointestinal bleeding. BMJ. Disponible en: https://casereports.bmj.com/content $/ 2015 /$ bcr-2015-211764

6. Snover DC, Weigent CE, Sumner HW. Diffuse mucosal ganglioneuromatosis of the colon associated with adenocarcinoma. Am J Clin Pathol 1981; 75 (2): 225-229.

7. Fryer JP. Intestinal transplantation: an update. Curr Opin Gastroenterol 2005; 21 (2): 162-168.

8. Fryer JP. The current status of intestinal transplantation. Curr Opin Organ Transplant 2008; 13: 266-272. 